\title{
Assessment of Stone Protective Coatings with a Novel Eco-Friendly Encapsulated Biocide
}

\author{
Martina Zuena, Ludovica Ruggiero (D), Giulia Caneva (D), Flavia Bartoli (D), Giancarlo Della Ventura (D), \\ Maria Antonietta Ricci (D) and Armida Sodo *(D)
}

Citation: Zuena, M.; Ruggiero, L.; Caneva, G.; Bartoli, F.; Della Ventura, G.; Ricci, M.A.; Sodo, A. Assessment of Stone Protective Coatings with a Novel Eco-Friendly Encapsulated Biocide. Coatings 2021, 11, 1109. https://doi.org/10.3390/ coatings11091109

Academic Editor: Giorgos Skordaris

Received: 28 July 2021

Accepted: 12 September 2021

Published: 14 September 2021

Publisher's Note: MDPI stays neutral with regard to jurisdictional claims in published maps and institutional affiliations.

Copyright: (c) 2021 by the authors. Licensee MDPI, Basel, Switzerland. This article is an open access article distributed under the terms and conditions of the Creative Commons Attribution (CC BY) license (https:/ / creativecommons.org/licenses/by/ $4.0 /)$.
Dipartimento di Scienze, Università degli Studi "Roma Tre", Via della Vasca Navale 84, 00146 Roma, Italy; martina.zuena@uniroma3.it (M.Z.); ludovica.ruggiero@uniroma3.it (L.R.); giulia.caneva@uniroma3.it (G.C.); flavia.bartoli@uniroma3.it (F.B.); giancarlo.dellaventura@uniroma3.it (G.D.V.); mariaantonietta.ricci@uniroma3.it (M.A.R.)

* Correspondence: armida.sodo@uniroma3.it

\begin{abstract}
The conservation of stone monuments is a constant concern due to their continuous weathering, in which biofouling plays a relevant role. To enhance the effectiveness of biocidal treatments and to avoid environmental issues related to their possible toxicity, this research aims at formulating and characterizing a coating charged with an eco-friendly biocide and showing hydrophobic properties. For this purpose, zosteric sodium salt-a natural biocide product-has been encapsulated into two silica nanocontainers and dispersed into a tetraethoxysilane-based (TEOS) coating also containing $\mathrm{TiO}_{2}$ nanoparticles. The coatings were applied on four different types of stone: brick, mortar, travertine, and Carrara marble. The effectiveness of the coating formulations and their compatibility concerning the properties of coated stones were assessed. The results showed that all coatings conferred a hydrophobic character to the substrate, as demonstrated by the increase of the static contact angle and the reduction in the capillary water absorption coefficient. The transmission of water vapor of the natural stones was preserved as well as their natural aspect. Furthermore, the coatings were homogeneously distributed on the surface and crack-free. Therefore, the protective capability of the coatings was successfully demonstrated.
\end{abstract}

Keywords: stone protection; monument biodeterioration; Si nanocontainers; antifouling; zosteric sodium salt; $\mathrm{TiO}_{2}$ nanoparticles

\section{Introduction}

Stone materials are widely used in architecture and artistic objects due to their durability, esthetics, availability, and easy manufacturing. Nevertheless, weathering processes may determine mechanical and esthetical concerns over time.

The most important weathering processes are connected to water penetrating the stone by the capillary rise or rainfall [1-4]. In particular, the presence of water may promote microorganism proliferation. This phenomenon is known as biodeterioration in the field of cultural heritage $[4,5]$ and more widely as fouling. Its incidence depends on the structural and textural features of the stones, as well as on environmental factors [5-9].

Biodeterioration phenomena are usually treated by applying biocidal solutions on the stone, which can be potentially harmful since they often involve toxic or polluting substances $[8,10]$. The research on less toxic compounds and natural biocides has been widely developed in the last few years [11-15]. A common approach is the preservation of the artifacts by applying a coating that inhibits the microorganism colonization. Several biocides have been tested so far after dispersion into coating formulations. However, the main issue of these attempts is their short-lived antifouling action due to quick release and possible deterioration of the active compound. Furthermore, the use of a large amount of biocide, required to preserve the biocide function for longer time, is dangerous for the environment and human health. To overcome these issues, we studied the formulation 
of a novel coating based on the encapsulation of an eco-friendly biocide into proper nanocontainers. This strategy helped to reduce the biocide quantity and protracting its efficacy over time, thanks to a controlled release. The proposed coating combined hydrophobic and biocide properties. This was achieved by adopting a natural biocide product-the zosteric sodium salt (ZS) in order to increase the coating eco-compatibilityand dispersing it into a water repellent coating [16-18].

The zosteric acid or p-(sulfo-oxy) cinnamic acid (ZS, Figure 1), naturally found in Zostera marina, or eelgrass, presents an antifouling capability due to the sulfate ester group [19-24]. This antifoulant does not kill microorganisms but avoids their adhesion on cell surfaces at non-toxic concentrations.<smiles>O=C(O)/C=C/c1ccc(OS(=O)(=O)O)cc1</smiles>

Figure 1. Structure formula of zosteric acid.

In our previous work, we encapsulated ZS into two different silica nanocontainers and we studied their release properties $[25,26]$. A preliminary in-vitro efficacy of encapsulated ZS against some common biodeteriogens has already been evaluated [26,27] and other experiments are currently ongoing, directly on site. Afterward, we dispersed the loaded nanodevices in a multifunctional TEOS-based coating also containing $\mathrm{TiO}_{2}$ nanoparticles as photocatalytic agents. This formulation was based on our previous experience, where a commercial biocide, 2-mercaptobenzothiazole, was employed. We designed and characterized the composite coating on a glass slide, to evaluate its best composition in terms of optical properties and lack of cracking [28]. Subsequently, we applied this coating on stone supports [29]. In the present paper, we report laboratory tests on the same coating formulation, loaded with a different green biocide, to investigate its influence on the microstructural properties and visual aspect of brick, mortar, travertine, and Carrara marble, which are widely used in the Cultural Heritage field.

\section{Materials and Methods}

\subsection{Substrates}

Experiments were carried out on both natural and man-made stones used in buildings and monuments; namely, red fired brick, a natural hydraulic lime-based mortar, travertine, and Carrara marble. All samples were obtained with the dimensions of $5 \times 5 \times 1 \mathrm{~cm}^{3}$. The red-fired brick (BR) was acquired from a local wholesale, which uses traditional methods. The lime-based mortar (MO) was obtained from the same local wholesale by using a mixture of 1:2 of natural hydraulic lime, NHL 5 (Saint Astier), and standard river sand as aggregate (size $<4 \mathrm{~mm}$ ). Water was added in a proper quantity to obtain a good workability of the mixture. The mortar samples were cured for 28 days at room conditions $\left(\mathrm{RH} \%=50 \%, 20^{\circ} \mathrm{C}\right)$. Carrara marble (MA) and travertine (TR)-extracted in quarries located in Carrara and Tivoli, respectively-underwent an aging process to increase their porosity. The adopted procedure [30] required two heating-cooling cycles: heating to $600{ }^{\circ} \mathrm{C}$, followed by storage in water overnight at room conditions $\left(\mathrm{RH} \%=50 \%, 20^{\circ} \mathrm{C}\right)$.

\subsection{Composition and Application of Coatings}

Two different silica nanocontainers, namely silica nanocapsules (NC) and silica mesoporous nanoparticles (MNP) loaded with $\mathrm{ZS}$, were synthesized according to the procedure reported in [31]. Acetyltrimethylammonium bromide (CTAB, Aldrich, St. Louis, MO, USA), ammonia solution ( $\mathrm{NH}_{3}$ aq. 30\%, Aldrich, St. Louis, $\mathrm{MO}$, USA), diethyl ether $\left(\mathrm{Et}_{2} \mathrm{O}\right.$, Aldrich, St. Louis, MO, USA), tetraethoxysilane (TEOS, Aldrich, St. Louis, MO, USA) were used without any further purification. ZS was synthesized from trans-4-hydroxycinnamic 
and the sulfur trioxide pyridine complex, according to the procedure reported in [22]. Some properties of the obtained loaded nanocontainers are reported in Table 1.

Table 1. Properties of silica nanocontainers loaded with ZS.

\begin{tabular}{cccc}
\hline Nanocontainer & Morphology & Size (nm) & $\begin{array}{c}\text { Loading Capability } \\
\text { (Weight \%) }\end{array}$ \\
\hline NC & Spherical & $170 \pm 20$ (diameter) & 2.1 \\
MNP & Rods & $100-1000$ (length) & 7.8 \\
\hline
\end{tabular}

These nanoparticles were dispersed in a coating, prepared following [32] and [28] by using TEOS, ethanol (Carlo Erba Reagents S.r.l., Cornaredo, Italy), poly(dimethylsiloxane) hydroxylterminated (PDMS-OH, Sigma-Aldrich, St. Louis, MO, USA), and n-octylamine (Sigma-Aldrich, St. Louis, MO, USA) as a non-ionic surfactant, without any further purification. The synthesis protocol required the following mole ratio: 1TEOS/16Ethanol $/ 10 \mathrm{H}_{2} \mathrm{O} / 0.04 \mathrm{PDMS}-\mathrm{OH} / 0.004 \mathrm{n}$ octylamine. The addition of PDMS-OH and n-octylamine to the original recipe reduced coating cracking and improved its adhesion to the stone surface as previously reported [28,29,33-35]. In fact, since TEOS is a silicate product, it is mostly compatible with silicate stones (brick in the present study) since it converts into amorphous silica once the polymerization process has been completed. In the case of carbonate stones (mortar, travertine and Carrara marble), the bonding between the carbonate stone and the silica gel is only physical $[36,37]$.

In addition to the biocide-loaded nanocontainers, $\mathrm{TiO}_{2}$ nanoparticles (anatase, SigmaAldrich) were also added. A total nanoparticle concentration of $0.1 \% w / w$ was used to obtain optimal results in terms of optical properties and lack of cracking, according to previous studies [28].

A coating without nanoparticles (Si_Control) was also applied as control, in addition to the investigated TEOS-based coatings containing $\mathrm{TiO}_{2}$ nanoparticles and loaded with silica nanocapsules $\left(\mathrm{Si}_{-} \mathrm{TiO}_{2}-\mathrm{NC}\right)$ or mesoporous nanoparticles $\left(\mathrm{Si}_{-} \mathrm{TiO}_{2}-\mathrm{MNP}\right)$.

An overview of the analyzed samples and the relative characterization techniques is reported in Table 2.

Table 2. Analyzed samples and applied characterization techniques.

\begin{tabular}{ccc}
\hline Sample & Description & Techniques \\
\hline $\mathrm{NT}$ & Untreated samples & SCA, CM, WAC, WVP, OSR, SEM, PT \\
$\mathrm{Si} \_$Control & Coating without nanoparticles & SCA, CM, WAC, WVP, OSR, SEM, PT \\
$\mathrm{Si} \_\mathrm{TiO}_{2}-\mathrm{NC}$ & Coating with $\mathrm{TiO}_{2}$ nanoparticles and loaded silica nanocapsules & SCA, CM, WAC, WVP, OSR, SEM, PT \\
$\mathrm{Si} \mathrm{TiO}_{2}-\mathrm{MNP}$ & Coating with $\mathrm{TiO}_{2}$ nanoparticles and loaded silica mesoporous & SCA, CM, WAC, WVP, OSR, SEM, PT \\
$\mathrm{Si}_{-} \mathrm{TiO}_{2}$ & nanoparticles & PT \\
\hline
\end{tabular}

The coatings were applied by brush until saturation, i.e., the condition for which the stone surface remains wet for more than $1 \mathrm{~min}$, to replicate a real application procedure on monuments. A total of 16 brushes were adopted for bricks and mortars, 4 for travertine, and 6 for Carrara marble, due to their different porosity and consequent absorption rate. The coatings were applied on the largest surface $\left(5 \times 5 \mathrm{~cm}^{2}\right)$ for all laboratory tests except absorption of water through capillarity, for which a smaller surface was treated $(5 \times 1$ $\mathrm{cm}^{2}$ ). About 1 week was required for coating solidification through polymerization and water evaporation, leaving the samples at laboratory conditions $\left(\mathrm{RH} \%=50 \%, 20^{\circ} \mathrm{C}\right)$. For all stones and treatments, the amount of the applied product—-that is, the sample weight difference before and right after the coating application —and the amount of retained product - that is, the sample weight difference before the coating application and one week after-are reported in Tables S1 and S2 in Supplementary Materials, respectively. 


\subsection{Assessment of Coated Stone Performance and Compatibility}

\subsubsection{Static Contact Angle (SCA)}

The wettability of the stone surface, as well as the hydrophobic effect of coatings, were evaluated by measuring the static contact angle before and after the application of the coating on the same sample. A total of 10 water droplets $(\sim 5 \mu \mathrm{L}$ each) were deposited on the stone surface at room temperature. The right and left angles observed between the water droplets and the stone surface after $10 \mathrm{~s}$ were captured by a high-resolution camera and then elaborated by computer analysis by using the AnalySIS Pro ${ }^{\circledR} 32$ software (Soft Imaging System GmbH, Münster, Germany). The results are expressed as the average of all droplets on each surface.

\subsubsection{Colorimetric Measurements (CM)}

The color of the selected stone specimen before and after the application of the coatings was measured by using an Eoptis CLM-194 portable colorimeter (Trento, Italy) following NORMAL 43/93 [38]. The obtained values are expressed in the CIEL $a^{*} b^{*}$ color space, where three parameters determine the color location: $L^{*}$ indicates the lightness $(0=$ absolute black, $100=$ absolute white), and $a^{*}$ and $b^{*}$ are the red/green and yellow /blue coordinates, respectively, with $\mathrm{a}^{*}<0$ red and $\mathrm{a}^{*}>0$ green, $b^{*}<0$ blue and $b^{*}>0$ yellow. The total color difference $\left(\Delta E^{*}\right)$ between untreated and treated samples was calculated according to the Equation (1):

$$
\Delta E=\sqrt{\Delta L^{* 2}+\Delta a^{* 2}+\Delta b^{* 2}}
$$

For each stone, three samples were analyzed before and after the application of the coatings. Three points for each specimen were measured.

\subsubsection{Water Absorption through Capillarity (WAC)}

The water absorption through capillarity was measured by following the normative UNI EN 15801:2010 [39]. The studied samples were previously dried at $60{ }^{\circ} \mathrm{C}$ until a constant mass, $m_{0}$, was observed. Then, they were placed on a filter paper saturated with distilled water and their weight, $m_{\mathrm{i}}$, was monitored at specific time intervals. Both the amount of absorbed water $(Q)$ and the capillary water absorption coefficient (CWAC) were calculated. The first parameter, expressed as $\left(\mathrm{kg} / \mathrm{m}^{2}\right)$, was obtained by the following Equation (2):

$$
Q=\left[\left(m_{\mathrm{i}}-m_{0}\right) / A\right]
$$

where $A$ is the surface in contact with the filter paper. The CWAC was calculated by linear fitting on the initial slope of $Q$ versus the square root of time. The analysis was performed on the same sample before and after treatment. Three samples were used for each stone.

\subsubsection{Water Vapor Permeability (WVP)}

The water vapor permeability, expressed as the water vapor permeability coefficient (g), was measured by following the standard DIN 52615 [40]. Stone specimens were placed on a metal cup containing a saturated aqueous solution of $\mathrm{KNO}_{3}$ in order to reach an internal humidity of $93 \%$ [41], and weighted every $24 \mathrm{~h}$, until weight stabilization. The test was performed on the same sample, treated and untreated. Triplicates of specimens were used for all types of stone, and the average results are reported.

\subsubsection{Optical Surface Roughness (OSR)}

Measurements of optical surface roughness were performed to evaluate changes in the surface morphology by using a confocal Leica DCM 3D optical profilometer (Leica Microsystems, Wetzlar, Germany). For this analysis, the roughness parameter $R z$ was considered; it refers to the average differences, within a sample length, between the largest peak height and the largest peak valley depth $[42,43]$. For each sample, three datasets were analyzed on both treated and untreated surfaces. A total of 500 profiles were acquired in the $\mathrm{Z}$ direction within an analyzed area of $5 \times 5 \mathrm{~mm}^{2}$ with a resolution along the axis of $2.5 \mu \mathrm{m}$. 


\subsubsection{Scanning Electron Microscopy (SEM) Combined to Energy Dispersive Spectroscopy (SEM-EDS)}

A Zeiss Sigma 300 SEM (Oberkochen, Germany) coupled with an ETSE (EverhartThornley secondary electron) detector were used to acquire SEM images from fragments of $1 \times 1 \times 1 \mathrm{~cm}^{3}$ from both untreated and treated samples. Combined with OSR, this analysis allows us to evaluate changes in the morphology of the surface and also the distribution of the coating, both on the surface and in-depth. All specimens were previously sputter-coated with gold and placed on a double-sided adhesive carbon tape. The operating voltages and working distances were set according to the analyzed samples. To evaluate the distribution of the coating both on the surface and in-depth, X-ray fluorescence chemical maps of Ti for brick and mortar, and Si for travertine and Carrara marble, were acquired with a $60 \mathrm{~mm}^{2}$ Bruker high-resolution EDS detector (energy dispersive X-ray, Berlin, Germany). The investigated area was $\sim 2400 \mathrm{~m}^{2}$. Since the matrix of brick and mortar is rich in $\mathrm{Si}$, this element was not investigated for these two materials.

\subsubsection{Photocatalysis Testing (PT)}

The photocatalytic activity of the $\mathrm{TiO}_{2}$ nanoparticles inserted in the coatings was evaluated under laboratory conditions. Stone samples were covered with a solution of $1 \mathrm{mM}$ of methyl orange (Riedel-de Haën, Seelze, Germany) used as a staining compound, which was diluted in ethanol to allow quick evaporation of the liquid once applied on the stones. Due to the different absorption of the stones, $750 \mu \mathrm{L}$ were used for brick and mortar and $500 \mu \mathrm{L}$ for travertine and Carrara marble. Moreover, also untreated samples (NT) and those treated with the coating loaded with only titanium nanoparticles $\left(\mathrm{Si}_{-} \mathrm{TiO}_{2}\right)$ were investigated as control. The stone specimens were placed in a ventilated chamber under a $365 \mathrm{~nm}$ UV light (Osram vitalux, Berlin, Germany), located at a distance of $20 \mathrm{~cm}$, for $72 \mathrm{~h}$ [35]. The same colorimeter adopted to evaluate colorimetric changes on the surface due to the application of the coatings was used to estimate the photocatalytic degradation of methyl orange. The reference at time zero for the discoloration curves corresponds with the surfaces without methyl orange. The obtained data are reported after normalization to 100 .

\section{Results and Discussion}

To assess the protective capability of the treatment, we first evaluated its hydrophobicity and possible color changes of the stone surface after application.

Information on the hydrophobicity of the coated stone surface was obtained by the SCA evaluation. The minimum acceptable SCA for stone protection is $90^{\circ}$ [44]; the measured values for our treatments are reported in Table 3 . The results concerning untreated stones are not reported, since the drops were absorbed too fast by the stone surfaces, due to their hydrophilic behavior. The obtained data demonstrate a good hydrophobization of the surfaces after treatment with all coatings. This confirms the hydrophobic behavior of the formulation, also shown in our previous work [29]. The addition of $\mathrm{TiO}_{2}$ nanoparticles and silica nanocontainers lightly increased the hydrophobic effect for all stones, except for mortar. Indeed, in the latter case, the SCA did not sensibly change within the standard deviation. No relevant differences were recorded between the coatings charged with $\mathrm{NC}$ and MNP applied on all stones.

Colorimetric measurements must be performed to verify that no visible color change is observed after the application of coatings and that the natural aspect of the stone is preserved [45]. This means that the total color variation $\Delta E^{*}$ should not be $>5$ [46]. Data registered after coatings application are shown in Figure 2, while the results concerning the individual parameters, namely brightness $L^{*}$, red-green chromatic component $a^{*}$, and yellow-blue chromatic component $b^{*}$ are reported in Table S3, in Supplementary Materials. All the investigated coatings showed a $\Delta E^{*}<5$, with no relevant differences among empty coatings and charged ones. The presence of MNP caused a higher color change of the surface with respect to NC for brick and mortar. However, since the obtained values remained below the limit of $\Delta E^{*}=5$, this difference can be considered irrelevant. Therefore, 
we can assume that the presence of nanoparticles did not influence the visual aspect of the treated stones.

Table 3. SCA values for all treated stones.

\begin{tabular}{cccc}
\hline \multirow{2}{*}{ Sample } & \multicolumn{3}{c}{ Average SCA ( ${ }^{\circ}$ ) } \\
\cline { 2 - 4 } & Si_Control $_{1}$ & Si_TiO $_{\mathbf{2}}-\mathbf{N C}$ & Si_TiO $_{\mathbf{2}}$-MNP \\
\hline Brick & $125.7 \pm 4.7$ & $138.2 \pm 5.1$ & $137.6 \pm 4.5$ \\
Mortar & $127.7 \pm 3.5$ & $130.1 \pm 5.5$ & $124.1 \pm 4.3$ \\
Travertine & $118.9 \pm 5.1$ & $120.2 \pm 5.1$ & $130.3 \pm 1.9$ \\
Carrara Marble & $125.5 \pm 4.2$ & $142.1 \pm 3.3$ & $134.2 \pm 5.1$ \\
\hline
\end{tabular}

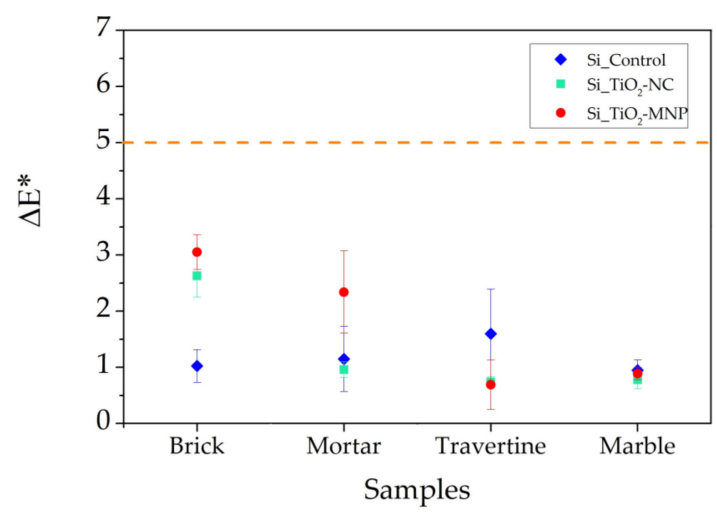

Figure 2. Mean global color variation $\Delta E^{*}$ between treated and untreated specimens in the $\operatorname{CIEL}^{*} a^{*} b^{*}$ color space. The thin vertical bars represent the mean standard deviation.

Transfer properties of liquid water and vapor were assessed by capillarity and water vapor permeability measurements, to investigate efficacy and harmlessness of the treatments. In fact, a proper protective coating should reduce the penetration of liquid water from outside and, likewise, should not prevent the outflow of water vapor towards the external side of the stone [47]. The capillary absorption kinetics of the stones, reported as a function of the square root of time $\left(\mathrm{s}^{1 / 2}\right)$, before and after the application of the coatings, are displayed in Figure 3. The slope of the first part of each curve refers to the capillary water absorption coefficient (CWAC), while the second part measures the reached saturation. The reduction in CWAC with respect to untreated stones is reported in Table 4. All untreated stones reached a different level of water saturation, within a distinctive time interval, according to their different porosity. In fact, the quantity of water absorbed by brick was higher $\left(\sim 17 \mathrm{Kg} / \mathrm{m}^{2}\right)$ than mortar $\left(\sim 10 \mathrm{Kg} / \mathrm{m}^{2}\right)$. At the same time, travertine and Carrara marble absorbed a similar amount of water $\left(\sim 2.3 \mathrm{Kg} / \mathrm{m}^{2}\right.$ and $\sim 1.7 \mathrm{Kg} / \mathrm{m}^{2}$, respectively), which is lower with respect to the previous supports. Moving to the treated samples, all coatings showed a considerable reduction in water absorption with respect to the bare stones. Notice that, in the case of brick and mortar, data for the treated samples refer to the right vertical scale in Figure $3 a-d$, as $Q$ changes by about one order of magnitude after treatment. For brick, a higher reduction of the initial slope of the curve is visible with $\mathrm{Si}_{-} \mathrm{TiO}_{2}-\mathrm{MNP}$ and $\mathrm{Si}_{-} \mathrm{TiO}_{2}-\mathrm{NC}$ compared with the Si_Control (Figure 3b). No differences are visible in the case of mortar (Figure $3 \mathrm{~d}$ ). 


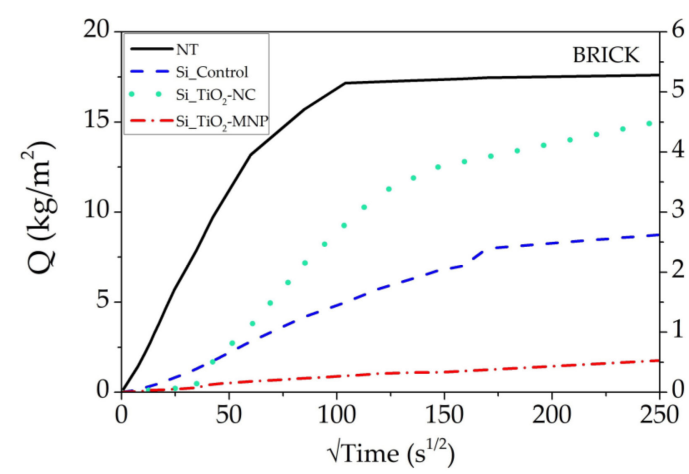

(a)

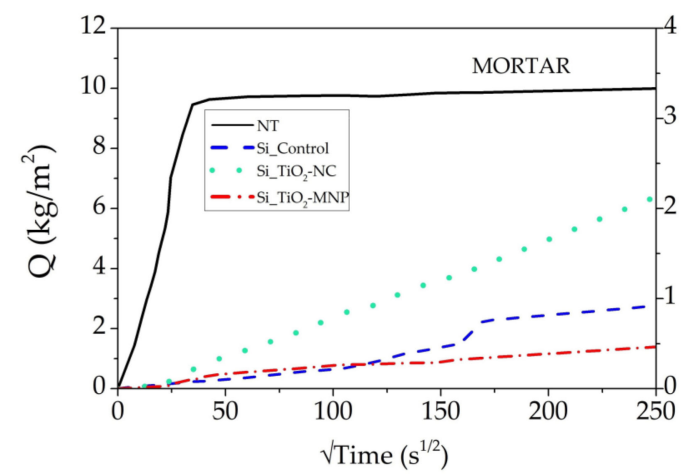

(c)

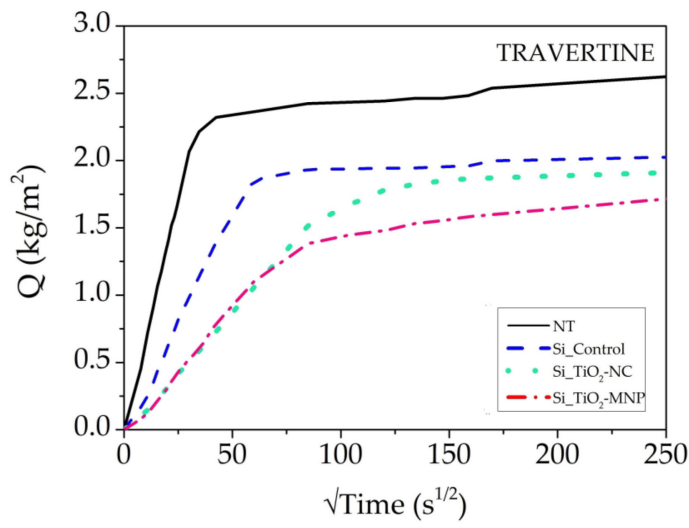

(e)

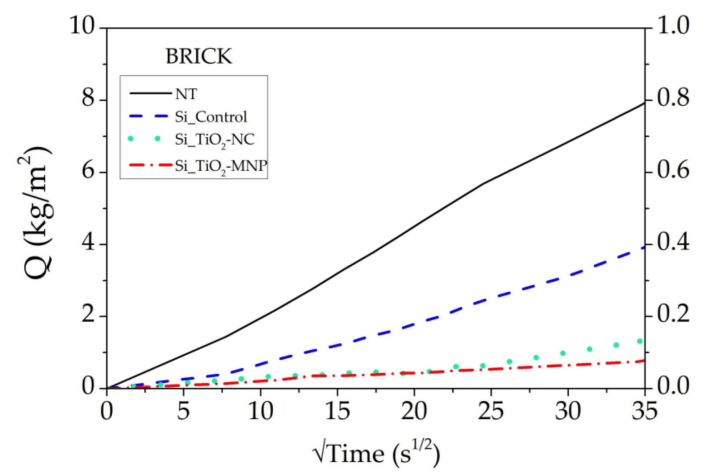

(b)

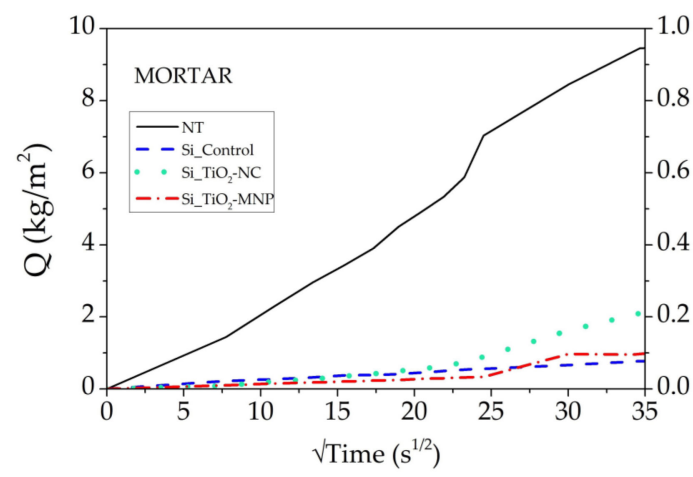

(d)

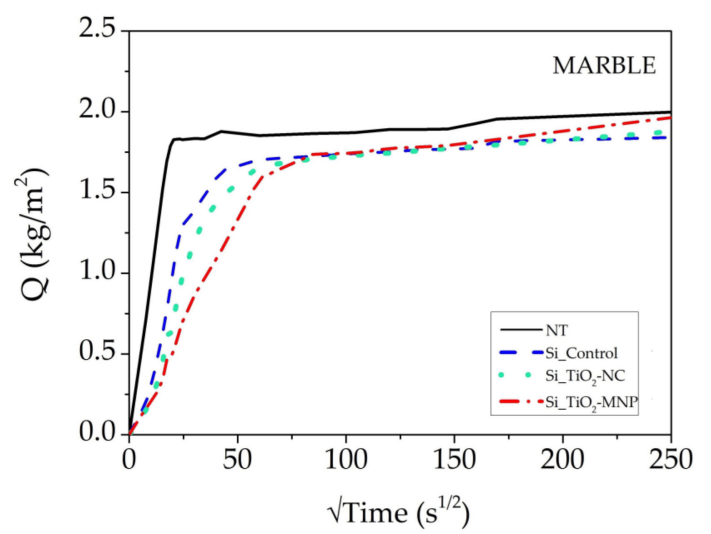

(f)

Figure 3. Plot of water absorption by capillarity test as a function of time: (a) brick, (b) brick (focus 0-35 $\mathrm{s}^{1 / 2}$ ), (c) mortar, (d) mortar (focus 0-35 $\mathrm{s}^{1 / 2}$ ), (e) travertine and (f) Carrara marble.

Table 4. Reduction (\%) of the capillary water absorption coefficient (CWAC), due to the coating.

\begin{tabular}{cccc}
\hline \multirow{2}{*}{ Sample } & \multicolumn{3}{c}{ Reduction in CWAC (\%) } \\
\cline { 2 - 4 } & Si_Control & Si_TiO $_{\mathbf{2}}-\mathbf{N C}$ & Si_TiO $_{\mathbf{2}}-\mathbf{M N P}$ \\
\hline Brick & 96.49 & 97.8 & 99.1 \\
Mortar & 98.97 & 98.9 & 99.5 \\
Travertine & 10.52 & 70.0 & 52.5 \\
Carrara marble & 46.25 & 47.8 & 68.8 \\
\hline
\end{tabular}

In the case of brick and mortar, the relative reduction of CWAC was higher than 95\% when compared to the untreated specimens (Table 4). A lower reduction was observed in the case of travertine and Carrara marble, possibly due to the lower number of applications (4 and 6, respectively). For travertine, the presence of nanoparticles had a higher effect on 
the reduction of CWAC. This may have been due to the size ratio between nanoparticles and stone porosity.

The measured water vapor permeability coefficient $(\mathrm{g})$ for all treated specimens did not sensibly change with respect to the case of the untreated samples, within the standard deviation (Figure 4). No relevant differences were registered between the presence of NC and MNP. Therefore, our results indicate that the tested coatings preserved the original water permeability of the materials.

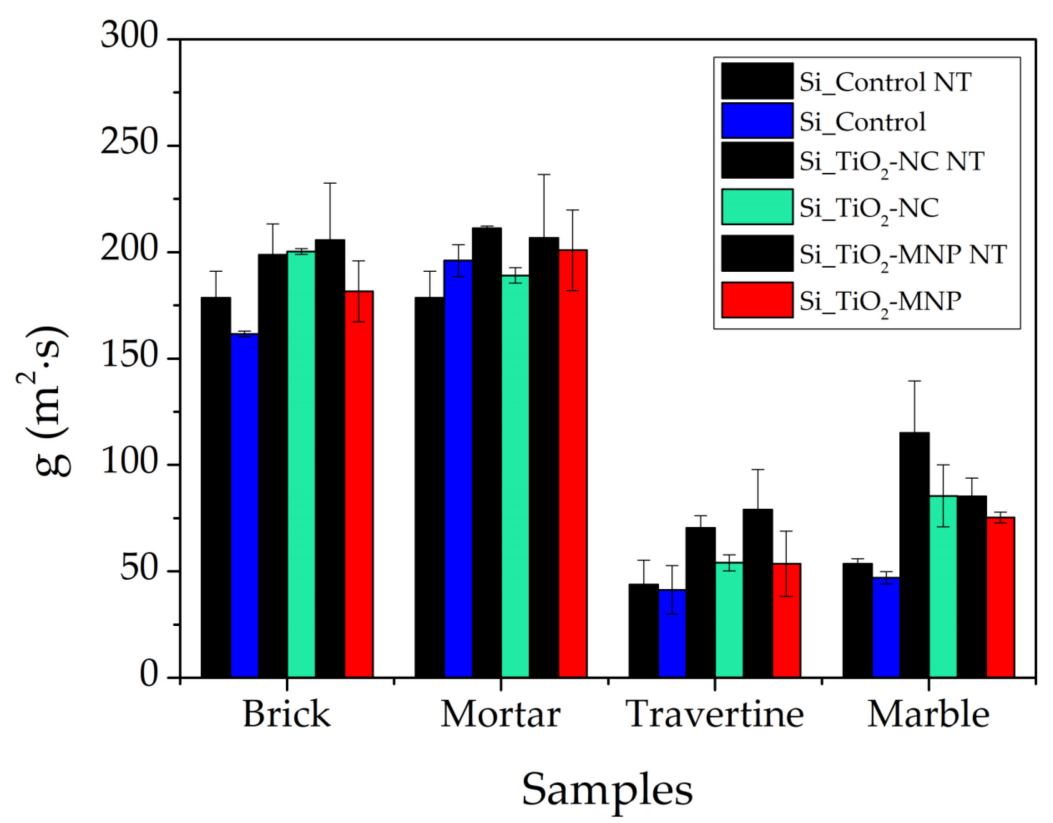

Figure 4. Mean water vapor permeability coefficient (g) shown for each stone before (black line on the left) and after (colored lines on the right) coatings application. The thin vertical black bar represents the mean standard deviation.

Roughness evaluations are reported in Figure S1, in Supplementary Materials. The results did not evidence particular trends of the roughness values after the application of the protective coatings, taking into account the significant standard deviations of $\mathrm{Rz}$.

SEM observations on coated stones showed compact films and no cracks for both empty coatings (Figure S2b,d,f,h, in Supplementary Materials) and nanoparticles loaded ones (Figure 5). The behavior concerning the presence of NC and MNP was similar, thus confirming the results obtained in our previous work [29]. To evaluate the distribution of the coating preparation and their depth of penetration, we acquired Ti distribution maps from brick and mortar, and Si ones from travertine and Carrara marble. These maps (Figures S3 and S4, in Supplementary Materials) demonstrated a homogeneous distribution of the coatings on all stone surfaces. Moreover, the protective characteristic of the coatings was demonstrated by the lack of penetration in depth evidenced by the absence of Ti and $\mathrm{Si}$ in cross-section measurements. 


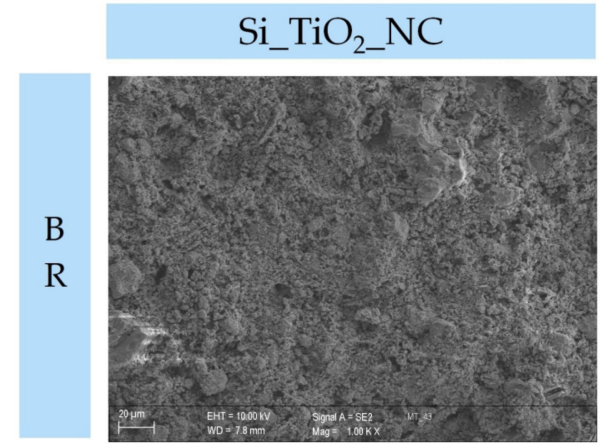

(a)

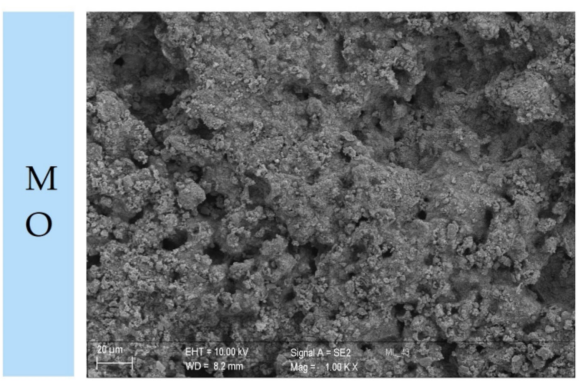

(c)

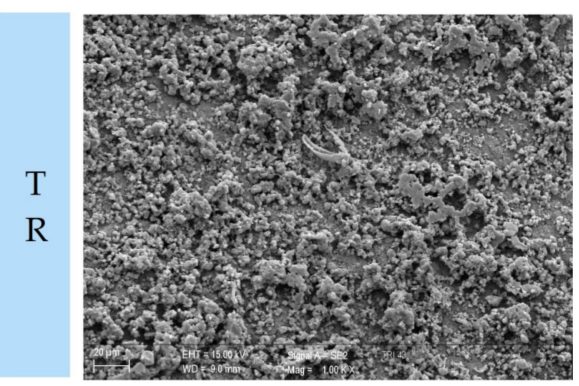

(e)

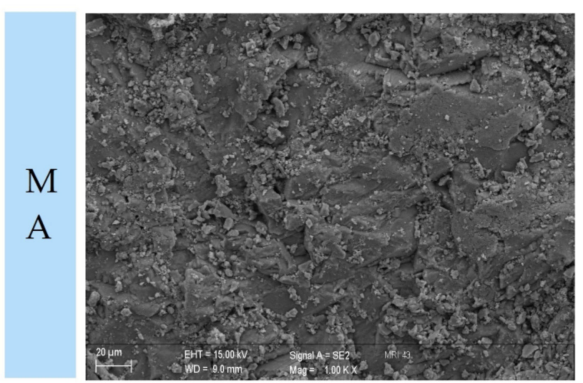

(g)

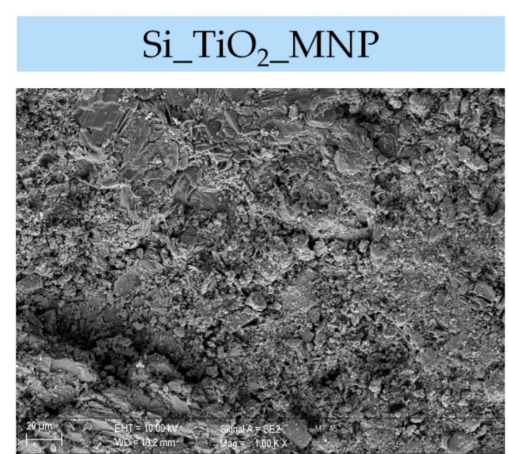

(b)

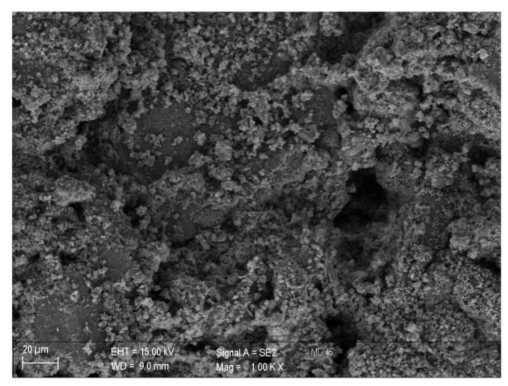

(d)

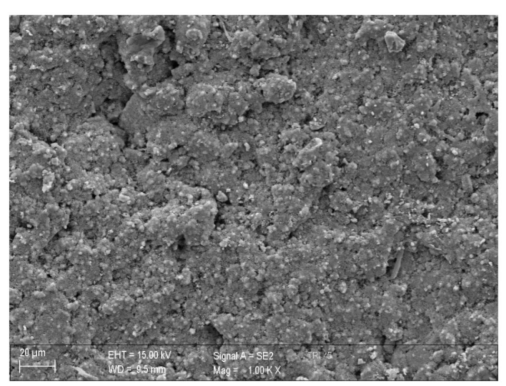

(f)

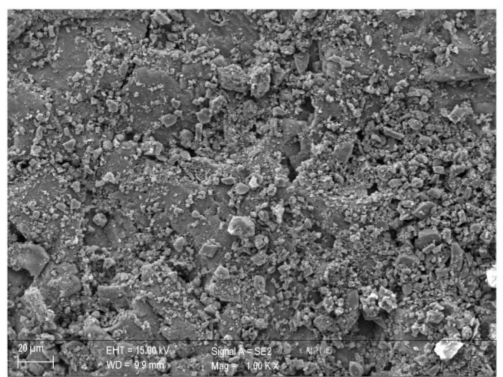

(h)

Figure 5. SEM images $(1000 \times)$ of coated stones treated with $\mathrm{Si}-\mathrm{TiO}_{2}-\mathrm{NC}$ and $\mathrm{Si}-\mathrm{TiO}_{2}-\mathrm{MNP}$ : $(\mathbf{a}, \mathbf{b})$ brick $(\mathrm{BR}),(\mathbf{c}, \mathbf{d})$, mortar $(\mathrm{MO}),(\mathbf{e}, \mathbf{f})$ travertine $(\mathrm{TR})$ and $(\mathbf{g}, \mathbf{h})$ Carrara marble (MA).

Aiming at evaluating the photocatalytic property of the $\mathrm{TiO}_{2}$ dispersed in the coating formulation, the degradation of methyl orange over time was examined under laboratory conditions. The discoloration of stones was reported as $\Delta E^{*}$ variation (Figure 6). In the case of brick, the color of the used stained compound was very similar to that of substrate. For this reason, the color differences among the consecutive measurements had larger uncertainties compared to those performed on the other supports. 


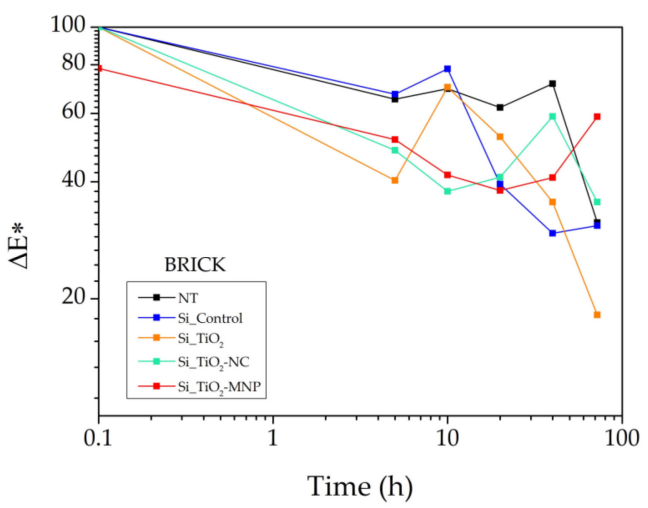

(a)

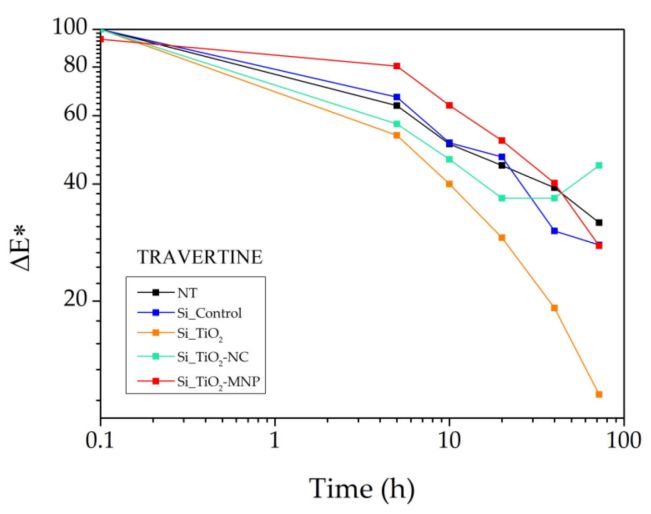

(c)

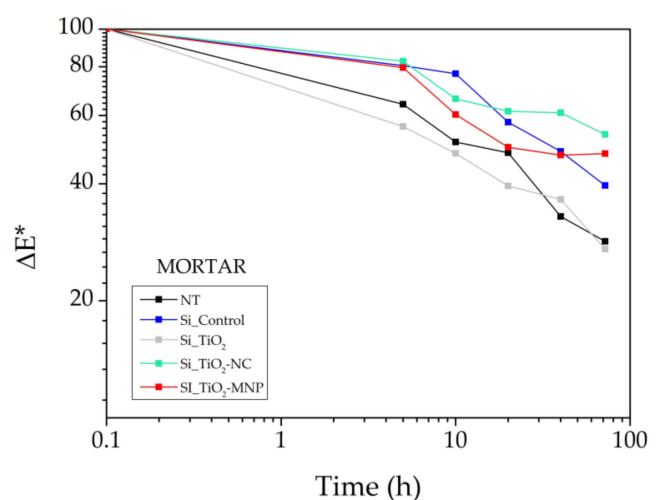

(b)

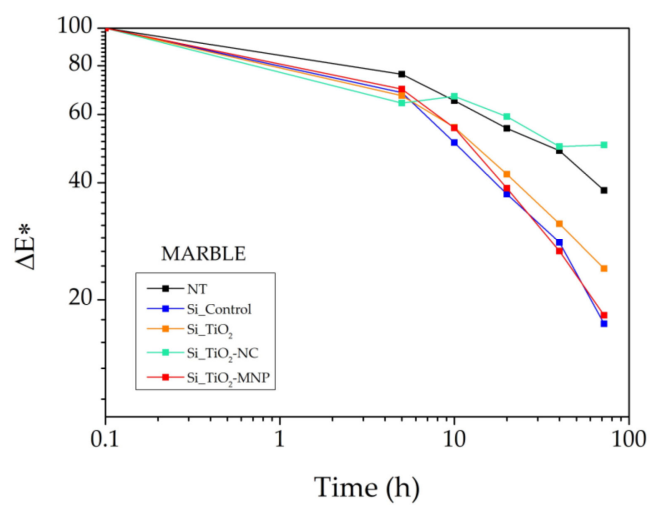

(d)

Figure 6. $\Delta E^{*}$ variation connected to the discoloration of treated and untreated stones previously stained with methyl orange and then irradiated with UV light for 72 h: (a) brick, (b) mortar, (c) travertine and (d) Carrara marble.

The results show that only for travertine, for which a lower number of coating application was performed, there was a higher photocatalytic activity of $\mathrm{Si}_{-} \mathrm{TiO}_{2}$ with respect to untreated samples and $\mathrm{Si}$ CControl. Both samples treated with $\mathrm{Si}-\mathrm{TiO}_{2}-\mathrm{NC}$ and $\mathrm{Si}_{-} \mathrm{TiO}_{2}-$ MNP showed worst results with respect to $\mathrm{Si}_{-} \mathrm{TiO}_{2}$, possibly because the presence of silica nanocontainers caused an entrapment of $\mathrm{TiO}_{2}$ nanoparticles, avoiding their correct contact with methyl orange. We noticed that the time evolution of $\Delta E^{*}$ was not exponential.

Such results indicate that the coatings presented in this work had mechanical and optical properties comparable between them and with the previous formulations containing a commercial harmful biocide [29]. These results were achieved despite the different particle dimensions and shape, and were consequent to the size and chemical properties of the encapsulated molecule. Further evaluation on a broad spectrum of activity against the potential occurring biodeteriogen are in progress.

\section{Conclusions}

The application of four different TEOS-based multifunctional coatings, containing silica nanocontainers loaded with an eco-friendly biocide $\mathrm{ZS}$ and $\mathrm{TiO}_{2}$ nanoparticle, confirmed their compatibility and effectiveness on the four tested stones, both natural and artificial. The presence of loaded-nanocontainers did not significantly alter the tested coating performances, while providing additional biocide efficacy. The presence of the coatings enhanced the stones' hydrophobicity and reduced water absorption through capillarity, with no evident difference between the two loaded nanocontainers. Importantly, our formulations did not significantly influence water vapor permeability and optical appearance of the stones. Thus, all fundamental requirements recommended to establish the effectiveness of a protective product for monument conservation were fulfilled by all presented coatings. We can, therefore, assume that these formulations are promising 
protective coatings even when stones with different mechanical and structural properties, as those tested here, are considered.

Supplementary Materials: The following are available online at https:/ / www.mdpi.com/article/10 .3390 / coatings11091109/s1, Table S1. Amount of product applied-the difference of sample weight before and right after coating application, Table S2. Amount of product retained-the difference of sample weight before and 1 week after coating application, Table S3. Brightness $L^{*}$, red-green chromatic component $a^{*}$ and yellow-blue chromatic component $b^{*}$ acquired before and after the coatings application on all stones, Figure S1. The roughness $R_{\mathrm{z}}$ estimated for both treated and untreated stones (NT), Figure S2. SEM images $(500 \times)$ of untreated samples and treated with empty coating: brick (BR) $(\mathrm{a}, \mathrm{b})$, mortar $(\mathrm{MO})(\mathrm{c}, \mathrm{d})$, travertine (TR) $(\mathrm{e}, \mathrm{f})$ and Carrara marble (MA) (g,h). NT: untreated; Si_Control, coating without nanoparticles, Figure S3. EDS-XFR Ti mapping acquired from brick and mortar: (a) brick treated with $\mathrm{Si}_{-} \mathrm{TiO}_{2}-\mathrm{NC}$, (b) brick treated with $\mathrm{Si}_{-} \mathrm{TiO}_{2}-\mathrm{MNP}$, (c) mortar treated with $\mathrm{Si}_{-} \mathrm{TiO}_{2}-\mathrm{NC}$ and (d) mortar treated with $\mathrm{Si}-\mathrm{TiO}_{2}-\mathrm{MNP}$, Figure S4. EDS-XFR Si mapping acquired from travertine and Carrara marble: (a) travertine treated with $\mathrm{Si}_{-} \mathrm{TiO}_{2}-\mathrm{NC}$, (b) travertine treated with $\mathrm{Si}_{-} \mathrm{TiO}_{2}-\mathrm{MNP}$, (c) Carrara Marble treated with $\mathrm{Si}_{-} \mathrm{TiO}_{2}-\mathrm{NC}$ and (d) Carrara marble treated with $\mathrm{Si}_{-}-\mathrm{TiO}_{2}-\mathrm{MNP}$.

Author Contributions: M.Z. carried out all the experiments, elaborated the data and wrote the paper. M.Z. and L.R. organized the experimental section, synthetized the coatings and finalized the photocatalysis proofs. G.D.V. supervised the SEM measurements. G.C., F.B. selected and provided the biocide, and performed biocidal tests. M.A.R. and A.S. supervised the project. All authors have read and agreed to the published version of the manuscript.

Funding: The authors acknowledge funding from Regione Lazio, Italy within the "SUPERARE" project (n. F86C18000650005) financed in the call "Gruppi di Ricerca" and "GRAL- Green and Longlasting stone conservation products" project (n. F85F21001710009) financed in the call "Progetto Gruppi di Ricerca 2020". The Grant of Excellence Departments, MIUR (ARTICOLO 1, COMMI 314-337 LEGGE 232/2016) is also greatly acknowledged.

Institutional Review Board Statement: Not applicable.

Informed Consent Statement: Not applicable.

Data Availability Statement: Data reported in this article are available on request.

Acknowledgments: LIME laboratory (Roma Tre University, Rome, Italy) is acknowledged for the possibility of carrying out some analyses. We acknowledge Sergio Lo Mastro for SEM analysis and Edoardo Bemporad, Daniele De Felicis, Riccardo Moscatelli, and Edoardo Rossi (Engineering department, Roma Tre University) for the technical support in the contact angle and optical surface roughness measurements. We acknowledge Laura Crociani for synthesizing the zosteric sodium salt (Institute of Condensed Matter Chemistry and Technologies for Energy (ICMATE), Consiglio Nazionale delle Ricerche (CNR), Padova, Italy).

Conflicts of Interest: The authors declare no conflict of interest.

\section{References}

1. Camuffo, D. Chapter 7-Atmospheric Water, Capillary Rise, and Stone Weathering; Elsevier: Amsterdam, The Netherlands, 2019.

2. Saad, A.; Guédon, S.; Martineau, F. Microstructural weathering of sedimentary rocks by freeze-thaw cycles: Experimental study of state and transfer parameters. Comptes Rendus-Geosci. 2010, 342, 197-203. [CrossRef]

3. Steiger, M.; Charola, E. Chapter 4-Weathering and deterioration. In Stone in Architecture; Siegesmund, R.S., Ed.; Springer: Berlin/Heidelberg, Germany, 2011; pp. 227-316.

4. Traversetti, L.; Bartoli, F.; Caneva, G. Wind-driven rain as a bioclimatic factor affecting the biological colonization at the archaeological site of Pompeii, Italy. Int. Biodeterior. Biodegrad. 2018, 134, 31-38. [CrossRef]

5. Caneva, G.; Bartoli, F.; Ceschin, S.; Salvadori, O.; Futagami, Y.; Salvati, L. Exploring ecological relationships in the biodeterioration patterns of Angkor temples (Cambodia) along a forest canopy gradient. J. Cult. Herit. 2015, 16, 728-735. [CrossRef]

6. Caneva, G.; Bartoli, F.; Savo, V.; Futagami, Y.; Strona, G. Combining statistical tools and ecological assessments in the study of biodeterioration patterns of stone temples in angkor (Cambodia). Sci. Rep. 2016, 6, 1-8. [CrossRef]

7. Sterflinger, K.; Piñar, G. Microbial deterioration of cultural heritage and works of art-Tilting at windmills? Appl. Microbiol. Biotechnol. 2013, 97, 9637-9646. [CrossRef] [PubMed]

8. Tiano, P. Biodeterioration of stone monuments a worldwide issue. Open Conf. Proc. J. 2016, 7, 29-38. [CrossRef] 
9. Casanova Municchia, A.; Bartoli, F.; Taniguchi, Y.; Giordani, P.; Caneva, G. Evaluation of the biodeterioration activity of lichens in the Cave Church of Üzümlü (Cappadocia, Turkey). Int. Biodeterior. Biodegrad. 2018, 127, 160-169. [CrossRef]

10. Urzi, C.; De Leo, F.; Krakova, L.; Pangallo, D.; Bruno, L. Effects of biocide treatments on the biofilm community in Domitilla's catacombs in Rome. Sci. Total Environ. 2016, 572, 252-262. [CrossRef] [PubMed]

11. Jeong, S.H.; Lee, H.J.; Kim, D.W.; Chung, Y.J. New biocide for eco-friendly biofilm removal on outdoor stone monuments. Int. Biodeterior. Biodegrad. 2018, 131, 19-28. [CrossRef]

12. Toreno, G.; Isola, D.; Meloni, P.; Carcangiu, G.; Selbmann, L.; Onofri, S.; Caneva, G.; Zucconi, L. Biological colonization on stone monuments: A new low impact cleaning method. J. Cult. Herit. 2018, 30, 100-109. [CrossRef]

13. Young, M.E.; Alakomi, H.L.; Fortune, I.; Gorbushina, A.A.; Krumbein, W.E.; Maxwell, I.; McCullagh, C.; Robertson, P.; Saarela, M.; Valero, J.; et al. Development of a biocidal treatment regime to inhibit biological growths on cultural heritage: BIODAM. Environ. Geol. 2008, 56, 631-641. [CrossRef]

14. Fidanza, M.R.; Caneva, G. Natural biocides for the conservation of stone cultural heritage: A review. J. Cult. Herit. 2019, 38, 271-286. [CrossRef]

15. Pinna, D. Coping with Biological Growth on Stone Heritage Objects: Methods, Products, Applications, and Perspectives; Apple Academic Press: Palm Bay, FL, USA, 2017; ISBN 978-354-077-3-405.

16. Colangiuli, D.; Calia, A.; Bianco, N. Novel multifunctional coatings with photocatalytic and hydrophobic properties for the preservation of the stone building heritage. Constr. Build. Mater. 2015, 93, 189-196. [CrossRef]

17. Ruffolo, S.A.; Ricca, M.; Macchia, A.; La Russa, M.F. Antifouling coatings for underwater archaeological stone materials. Prog. Org. Coat. 2017, 104, 64-71. [CrossRef]

18. Pinna, D.; Salvadori, B.; Galeotti, M. Monitoring the performance of innovative and traditional biocides mixed with consolidants and water-repellents for the prevention of biological growth on stone. Sci. Total Environ. 2012, 423, 132-141. [CrossRef]

19. Geiger, T.; Delavy, P.; Hany, R.; Schleuniger, J. Encapsulated Zosteric Acid Embedded in Poly [3- hydroxyalkanoate] CoatingsProtection against Biofouling. Polym. Bull. 2004, 52, 65-72. [CrossRef]

20. Boopalan, M.; Sasikumar, A. Studies on Biocide Free and Biocide Loaded Zeolite Hybrid Polymer Coatings on Zinc Phosphated Mild Steel for the Protection of Ships Hulls from Biofouling and Corrosion. Silicon 2011, 3, 207-214. [CrossRef]

21. Laabir, M.; Grignon-Dubois, M.; Masseret, E.; Rezzonico, B.; Soteras, G.; Rouquette, M.; Rieuvilleneuve, F.; Cecchi, P. Algicidal effects of Zostera marina L. and Zostera noltii Hornem. extracts on the neuro-toxic bloom-forming dinoflagellate Alexandrium catenella. Aquat. Bot. 2013, 111, 16-25. [CrossRef]

22. Villa, F.; Pitts, B.; Stewart, P.S.; Giussani, B.; Roncoroni, S.; Albanese, D.; Giordano, C.; Tunesi, M.; Cappitelli, F. Efficacy of Zosteric Acid Sodium Salt on the Yeast Biofilm Model Candida albicans. Microb. Ecol. 2011, 62, 584-598. [CrossRef]

23. Almeida, J.R.; Moreira, J.; Pereira, D.; Pereira, S.; Antunes, J.; Palmeira, A.; Vasconcelos, V.; Pinto, M.; Correia-da-Silva, M.; Cidade, H. Potential of synthetic chalcone derivatives to prevent marine biofouling. Sci. Total Environ. 2018, 643, 98-106. [CrossRef]

24. Cattò, C.; Dell'Orto, S.; Villa, F.; Villa, S.; Gelain, A.; Vitali, A.; Marzano, V.; Baroni, S.; Forlani, F.; Cappitelli, F. Unravelling the structural and molecular basis responsible for the anti-biofilm activity of zosteric acid. PLoS ONE 2015, 10, e0131519. [CrossRef] [PubMed]

25. Ruggiero, L.; Bartoli, F.; Fidanza, M.R.; Zurlo, F.; Marconi, E.; Gasperi, T.; Tuti, S.; Crociani, L.; Di Bartolomeo, E.; Caneva, G.; et al. Encapsulation of environmentally-friendly biocides in silica nanosystems for multifunctional coatings. Appl. Surf. Sci. 2020, 514, 145908. [CrossRef]

26. Ruggiero, L.; Crociani, L.; Zendri, E.; El Habra, N.; Guerriero, P. Incorporation of the zosteric sodium salt in silica nanocapsules: Synthesis and characterization of new fillers for antifouling coatings. Appl. Surf. Sci. 2018, 439, 705-711. [CrossRef]

27. Bartoli, F.; Zuena, M.; Sodo, A.; Caneva, G. The efficiency of biocidal silica nanosystems for the conservation of stone monuments: Comparative in vitro tests against epilithic green algae. Appl. Sci. 2021, 11, 6804. [CrossRef]

28. Ruggiero, L.; Fidanza, M.R.; Iorio, M.; Tortora, L.; Caneva, G.; Ricci, M.A.; Sodo, A. Synthesis and characterization of TEOS coating added with innovative antifouling silica nanocontainers and $\mathrm{TiO}_{2}$ nanoparticles. Frontiers 2020, 7, 1-11. [CrossRef]

29. Zuena, M.; Ruggiero, L.; Della Ventura, G.; Bemporad, E.; Ricci, M.A.; Sodo, A. Effectiveness and compatibility of nanoparticle based multifunctional coatings on natural and man-made stones. Coatings 2021, 11, 480. [CrossRef]

30. Haake, S.; Simon, S.; Favaro, M. The bologna cocktail-evaluation of consolidation treatments on monuments in france and italy after 20 years on natural aging. In Proceedings of the 10th International Congress on Deterioration and Conservation of Stone, Stockholm, Sweden, 27 June-2 July 2004; pp. 423-430.

31. Ruggiero, L.; Di Bartolomeo, E.; Gasperi, T.; Luisetto, I.; Talone, A.; Zurlo, F.; Peddis, D.; Ricci, M.A.; Sodo, A. Silica nanosystems for active antifouling protection: Nanocapsules and mesoporous nanoparticles in controlled release applications. J. Alloys Compd. 2019, 798, 144-148. [CrossRef]

32. Xu, F.; Li, D.; Zhang, Q.; Zhang, H.; Xu, J. Effects of addition of colloidal silica particles on TEOS-based stone protection using n-octylamine as a catalyst. Prog. Org. Coat. 2012, 75, 429-434. [CrossRef]

33. Kapridaki, C.; Maravelaki-Kalaitzaki, P. TiO2-SiO2-PDMS nano-composite hydrophobic coating with self-cleaning properties for marble protection. Prog. Org. Coat. 2013, 76, 400-410. [CrossRef]

34. Pinho, L.; Elhaddad, F.; Facio, D.S.; Mosquera, M.J. A novel $\mathrm{TiO}_{2}-\mathrm{SiO}_{2}$ nanocomposite converts a very friable stone into a self-cleaning building material. Appl. Surf. Sci. 2013, 275, 389-396. [CrossRef] 
35. Pinho, L.; Mosquera, M.J. Photocatalytic activity of $\mathrm{TiO}_{2}-\mathrm{SiO}_{2}$ nanocomposites applied to buildings: Influence of particle size and loading. Appl. Catal. B Environ. 2013, 134-135, 205-221. [CrossRef]

36. Scherer, G.W.; Wheeler, G.S. Silicate consolidants for stone. Key Eng. Mater. 2008, 391, 1-25. [CrossRef]

37. Sassoni, E.; Franzoni, E.; Pigino, B.; Scherer, G.W.; Naidu, S. Consolidation of calcareous and siliceous sandstones by hydroxyapatite: Comparison with a TEOS-based consolidant. J. Cult. Herit. 2013, 14, e103-e108. [CrossRef]

38. NORMAL 43/93 Misure Colorimetriche di Superfici Opache (Italian Normative on Stone Material_Colorimetric Measurement of Opaque Surfaces); Commissione Beni Culturali UNI NORMAL: Roma, Italy, 1993.

39. EN UNI 15801:2010 Conservation of Cultural Property-Test Methods-Determination of Water Absorption by Capillarity. 2010. Available online: https://standards.iteh.ai/catalog/standards/cen/06e6ae49-1d9a-4318-b79b-eae91a02091c/en-15801-2009 (accessed on 9 December 2009).

40. DIN 52615 Testing of Thermal Insulating Material. In Determination of Water Vapour Permeability of Construction and Insulating Materials; Deutsches Institut fur Normung E.V. (DIN): Berlin, Germany, 1987.

41. Greenspan, L. Humidity fixed points of binary saturated aqueous solutions. J. Res. Natl. Bur. Stand. Sect. A Phys. Chem. 1977, 81A 89. [CrossRef]

42. Hobson, T. Parameters and Definitions for Roundness and Surface Measurements; Taylor Hobson Ltd.: Leicester, UK, 2000.

43. Della Volpe, C.; Penati, A.; Peruzzi, R.; Siboni, S.; Toniolo, L.; Colombo, C. Combined effect of roughness and heterogeneity on contact angles: The case of polymer coating for stone protection. J. Adhes. Sci. Technol. 2000, 14, 273-299. [CrossRef]

44. Alvarez de Buergo Ballester, M.; Fort González, R. Basic methodology for the assessment and selection of water-repellent treatments applied on carbonatic materials. Prog. Org. Coat. 2001, 43, 258-266. [CrossRef]

45. Sasse, H.R.; Snethlage, R. Methods for the evaluation of stone conservation treatments. In Saving Our Cultural Heritage: The Conservation of Historic Stone Structure, Dahlem Workshop Reports; Baer, N.S., Snethlage, R., Eds.; Wiley \& Sons: Chichester, UK, 1997; pp. 223-243.

46. Delgado Rodrigues, J.; Grossi, A. Indicators and ratings for the compatibility assessment of conservation actions. J. Cult. Herit. 2007, 8, 32-43. [CrossRef]

47. Tsakalof, A.; Manoudis, P.; Karapanagiotis, I.; Chryssoulakis, I.; Panayiotou, C. Assessment of synthetic polymeric coatings for the protection and preservation of stone monuments. J. Cult. Herit. 2007, 8, 69-72. [CrossRef] 